\title{
Aplicação de ozônio contra Sitophilus zeamais e Tribolium castaneum em milho armazenado ${ }^{1}$
}

\author{
Adriano F. Rozado ${ }^{2}$, Lêda R. A. Faroni ${ }^{3}$, Wilfredo M. I. Urruchi ${ }^{4}$, Raul N. C. Guedes ${ }^{5}$ \& Juliana L. Paes ${ }^{6}$
}

\section{RESUMO}

Neste trabalho a meta principal foi avaliar a suscetibilidade dos adultos de Sitophilus zeamais (Motschulsky) (Coleoptera: Curculionidae) e Tribolium castaneum (Herbst) (Coleoptera: Tenebrionidae) e a qualidade fisiológica dos grãos de milho submetidos ao tratamento com ozônio, em diferentes camadas da massa de grãos, razão pela qual se construíram gaiolas de 3,0 cm de altura e 15,0 cm de diâmetro, em PVC, sendo o fundo e a tampa confeccionados em tecido do tipo organza; as gaiolas foram dispostas em recipientes cilíndricos, também em PVC, com $20 \mathrm{~cm}$ de diâmetro, $100 \mathrm{~cm}$ de altura e conexões para injeção e exaustão do gás ozônio. Tanto os insetos quanto os grãos de milho foram submetidos a uma atmosfera modificada com $50 \mathrm{mg} \mathrm{kg}^{-1}$ de ozônio injetado a um fluxo contínuo de 8,0 $\mathrm{L} \mathrm{min}^{-1}$ em conexão localizada na base (plenum) do recipiente, em diferentes períodos de exposição. A espécie que se mostrou mais susceptível foi a S. zeamais, independentemente da sua distribuição na massa de grãos. O menor período de exposição para controlar $95 \%$ dos adultos de $S$. zeamais e $T$. castaneum foi de 23,76 e 64,19 h, respectivamente, quando distribuídos próximos à injeção do gás ozônio; em geral, os tratamentos com atmosfera modificada com $50 \mathrm{mg} \mathrm{kg}^{-1}$ de ozônio não afetaram a qualidade fisiológica dos grãos de milho.

Palavras-chave: atmosfera modificada, insetos, fumigação

\section{Ozone application against Sitophilus zeamais and Tribolium castaneum on stored maize}

\begin{abstract}
The main goal of this work was to assess the susceptibility of adults of Sitophilus zeamais (Motschulsky) (Coleoptera: Curculionidae) and Tribolium castaneum (Herbst) (Coleoptera: Tenebrionidae) and the physiological quality of maize grains subjected to ozone treatment in different grain layers. PVC cages $3.0 \mathrm{~cm}$ high and $15.0 \mathrm{~cm}$ diameter were built with their bottom and cover closed with organza-like cloth; the cages were placed in cylindrical containers of PVC with $20 \mathrm{~cm}$ diameters and $100 \mathrm{~cm}$ heights and connections for injection and exhaustion of the ozone gas. Insects and maize grains were subjected to a modified atmosphere with $50 \mathrm{mg} \mathrm{kg}^{-1}$ ozone injected at a flow of $8.0 \mathrm{~L} \mathrm{~min}^{-1}$ through a connection at the bottom of the container (plenum) for different exposure times. Sitophilus zeamais was the most susceptible species regardless of its distribution in the grain mass. The exposure periods required to achieve $95 \%$ control of adults of $S$. zeamais and T. castaneum were 23.76 and $64.19 \mathrm{~h}$ respectively, when distributed near the ozone injection; overall the treatments with atmosphere modified by ozone injection at $50 \mathrm{mg} \mathrm{kg}^{-1}$ did not affect the physiological quality of maize grain.
\end{abstract}

Key words: modified atmosphere, insects, fumigation

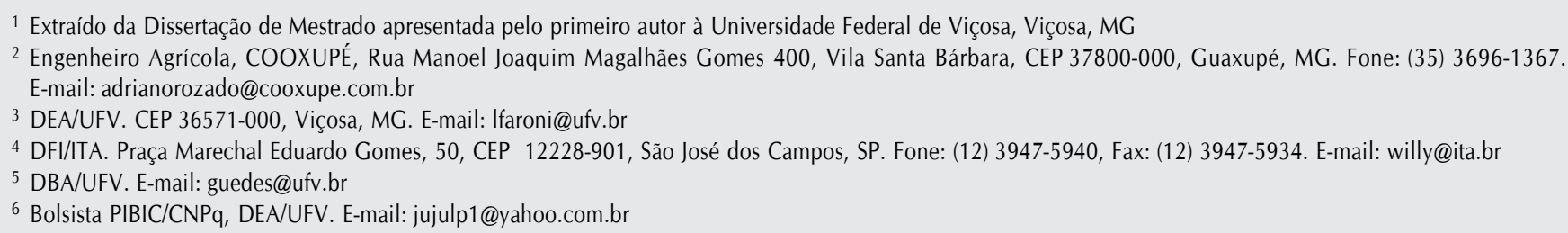




\section{INTRODUÇÃO}

O armazenamento de grãos é parte integrante do sistema de pré-processamento de produtos agrícolas; nesta fase os grãos são submetidos a fatores físicos, químicos e biológicos, que podem interferir na sua conservação e qualidade (Brooker et al., 1992). É visível a necessidade contínua de se proteger os produtos armazenados contra a deterioração evitando-se, desta forma, perdas de qualidade e quantidade durante o armazenamento, objetivando-se atender a um mercado cada vez mais exigente (Padin et al., 2002).

No âmbito agrícola, alguns estudos estão sendo desenvolvidos com o intuito de preservar a qualidade dos grãos e subprodutos armazenados.

Nos últimos anos, a utilização do ozônio tem-se expandido de forma considerável, nacional ou internacionalmente, em diferentes áreas de aplicação, como no tratamento de água potável, efluentes domésticos e industriais e processos de branqueamento de celulose, entre outros. Novos segmentos de aplicações de ozônio são desenvolvidos, principalmente nas áreas de processamento de alimentos e agricultura, com aprovação da FDA (Food and Drug Administration) dos Estados Unidos, para esta finalidade (Rice \& Graham, 2002).

Um manejo moderno de pragas em unidades armazenadoras e processadoras de grãos deve ser composto por medidas de controle eficazes, de baixo custo e com um mínimo de impacto ambiental, induzindo, desta forma, a geração de novas tecnologias e melhor manejo daquelas já existentes. Dentre as novas tecnologias no controle de pragas, o ozônio pode tornar-se uma alternativa ecologicamente correta e economicamente viável no âmbito da manutenção e preservação da qualidade dos produtos de origem vegetal.

Além do controle de insetos que o ozônio pode promover, outro parâmetro importante a ser observado quanto à sua utilização, é a qualidade do produto armazenado. Segundo Mendez et al. (2003), nenhum efeito negativo na qualidade e nas características nutricionais do trigo, milho e arroz foi verificado, quando submetidos a um tratamento com $50 \mathrm{mg}$ $\mathrm{kg}^{-1}$ de ozônio durante 30 dias.

$\mathrm{O}$ ozônio $\left(\mathrm{O}_{3}\right)$ é um poderoso agente oxidante que pode ser gerado no local, através de um processo de descarga elétrica (Kim et al.,1999); desta forma, sua utilização se torna atraente no controle de insetos e fungos em grãos armazenados, pelo fato de descartar a necessidade de manipulação, armazenamento ou eliminação dos recipientes de produtos químicos e, ainda, em virtude de possuir uma meia vida curta e de seu produto de degradação ser o oxigênio (Kells et al., 2001; Mendez et al., 2003).

Ante o exposto, foi meta deste trabalho avaliar a suscetibilidade dos adultos de $S$. zeamais e $T$. castaneum, e a qualidade fisiológica dos grãos de milho submetidos ao tratamento com ozônio, em diferentes camadas na massa de grãos.

\section{MATERIAL E MÉTODOS}

O presente estudo foi desenvolvido no Setor de Armazenamento e Pré-Processamento de Produtos Agrícolas do De- partamento de Engenharia Agrícola da Universidade Federal de Viçosa, MG.

Para os tratamentos, grãos de milho com padrão de classificação tipo 1, com teor de umidade em torno de 13\% (b.u.) foram distribuídos em recipientes cilíndricos, construídos em PVC, com $20 \mathrm{~cm}$ de diâmetro, $100 \mathrm{~cm}$ de altura e conexões para injeção e exaustão de gás; a $10 \mathrm{~cm}$ da base do recipiente colocou-se uma tela metálica para sustentação dos grãos e formação de um plenum, para melhor distribuição do gás.

Para avaliar a eficácia do ozônio como fumigante, grãos de milho infestados com adultos de $S$. zeamais $e$ T. castaneum, obtidos de criação contínua em câmara climática do tipo B.O.D., foram distribuídos em gaiolas de 3,0 cm de altura e 15,0 cm de diâmetro, também em PVC, sendo o fundo e a tampa confeccionados em tecido do tipo organza; essas gaiolas foram dispostas nos recipientes cilíndricos em diferentes camadas da massa de grãos (sobre o plenum, mediana e superior) e submetidas a uma atmosfera modificada com $50 \mathrm{mg} \mathrm{kg}^{-1}$ de ozônio e ar atmosférico (testemunha), em diferentes períodos de exposição estimando-se, assim, os tempos letais (TL) para 50 e 95\% da população de cada espécie. Os dados de mortalidade foram submetidos à análise de Probite, através do programa SAS ${ }^{\circledR}$ for Windows ${ }^{\mathrm{TM}}$, versão 6.12, para avaliação do efeito dos diferentes períodos de exposição gerando, desta forma, as curvas de mortalidade.

Para avaliar o efeito do ozônio na qualidade do produto, grãos de milho com padrão de classificação tipo 1 , sadios e intactos, com teor de umidade em torno de 13\% (b.u.), foram distribuídos em gaiolas de 3,0 cm de altura e 15,0 cm de diâmetro, em PVC, em que o fundo e a tampa foram confeccionados em tecido do tipo organza; as gaiolas, contendo os grãos de milho, foram dispostas em diferentes camadas da massa de grãos (sobre o plenum, mediana e superior) e submetidas a uma atmosfera modificada com $50 \mathrm{mg} \mathrm{kg}^{-1}$ de ozônio e com ar atmosférico (testemunha), em diferentes períodos de exposição. Tanto os grãos submetidos ao tratamento com ozônio quanto aqueles tratados com ar atmosférico foram submetidos a testes de germinação, condutividade elétrica e teor de umidade; desta forma e por meio de comparação entre os tratamentos, avaliou-se o efeito da fumigação com ozônio na qualidade dos grãos. Os dados de qualidade do milho foram submetidos a modelos de regressão e as análises realizadas para cada nível da massa de grãos.

Em todo o experimento, o ozônio e o ar atmosférico foram injetados a um fluxo contínuo de $8,0 \mathrm{~L} \mathrm{~min}^{-1}$, em conexão localizada na base (plenum) do recipiente e a temperatura da massa de grãos mantida em $25{ }^{\circ} \mathrm{C}$, sendo esta monitorada por meio de um sistema de aquisição e armazenamento de dados, denominado 1-wire. Em todos os ensaios se utilizaram seis recipientes cilíndricos injetando-se, em três deles, ozônio e, nos outros, ar atmosférico (testemunha).

\section{RESULTADOS E DISCUSSÃO}

As Figuras 1A, 1B e 1C mostram as curvas tempo-resposta para adultos de $S$. zeamais e de T. castaneum, submetidos a $50 \mathrm{mg} \mathrm{kg}^{-1}$ de ozônio, distribuídos sobre o plenum, 
nas camadas mediana e superior da massa de grãos. A eficácia no controle dos insetos-praga $S$. zeamais e $T$. castaneum foi influenciada pelo período de exposição e pela distribuição dos insetos na massa de grãos.

À medida que se aumentou o período de exposição, houve uma elevação da mortalidade. O período de exposição aumentou com o distanciamento do ponto de injeção do gás.
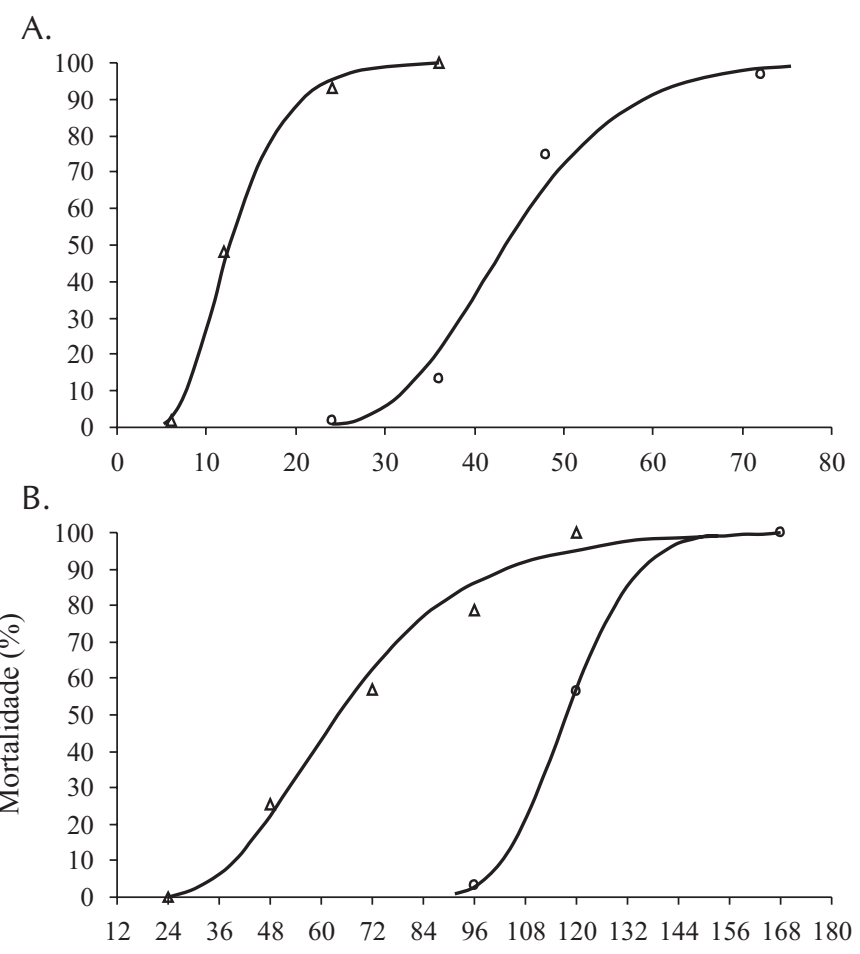

C.

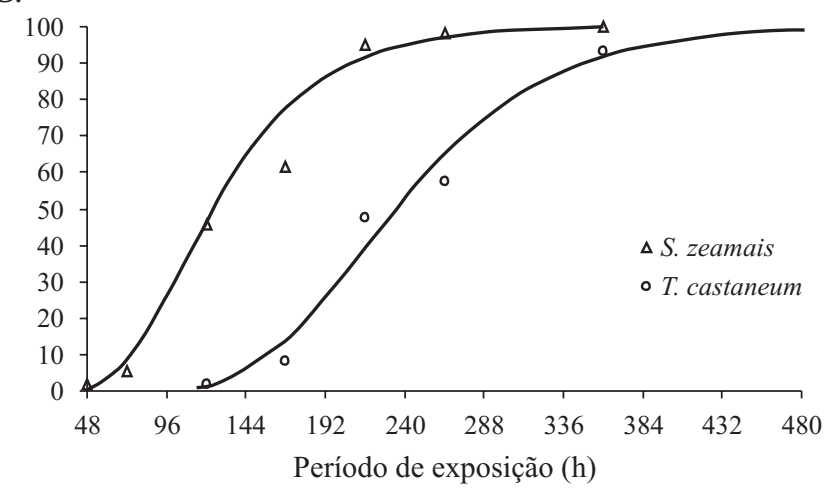

Figura 1. Tempo necessário para controlar adultos de $S$. zeamais e de $T$. castaneum, distribuídos sobre o plenum $(A)$, na camada mediana $(B)$ e na superfície (C)
Segundo Strait (1998) e Kells et al. (2001), o processo de fumigação com ozônio apresenta duas fases distintas: na primeira, ocorre uma rápida degradação do ozônio e, na segunda, o ozônio flui livremente pelo grão com pouca degradação. O tempo letal para os adultos de $S$. zeamais foi menor que o obtido para T. castaneum (Figuras 1A, 1B e 1C); em todas as distribuições dos insetos na massa de grãos, este resultado é confirmado por Kells et al. (2001) que, estudando a eficácia do ozônio como fumigante, na concentração de $50 \mathrm{mg} \mathrm{kg}^{-1}$, no controle de T. castaneum e de S. zeamais, obtiveram eficácia de 92 e 100\%, respectivamente, em três dias, em uma aplicação localizada, ou seja, próxima ao ponto de injeção do ozônio.

Para avaliar se os grãos submetidos ao tratamento com $50 \mathrm{mg} \mathrm{kg}^{-1}$ de ozônio apresentariam diferenças significativas em relação àqueles tratados com ar atmosférico, os dados obtidos nos testes de qualidade dos grãos foram submetidos a análise de regressão até o modelo de terceiro grau. Não houve diferença significativa entre os tratamentos, com exceção apenas para a condutividade elétrica da solução contendo os grãos distribuídos na camada superior da coluna. Apresentamse, na Tabela 1, os valores médios do teor de umidade, de condutividade elétrica e do potencial de germinação dos grãos submetidos aos tratamentos com $50 \mathrm{mg} \mathrm{kg}^{-1}$ de ozônio e com ar atmosférico, distribuídos ao longo da coluna.

Conforme a Figura 2, o aumento no tempo de exposição à atmosfera modificada com ozônio causou uma elevação da condutividade elétrica, a partir de 264 h, na solução que continha os grãos de milho distribuídos na camada superior da coluna. A elevação da condutividade elétrica indica a liberação de íons para a solução aquosa devido a lixiviação de eletrólitos teciduais (Vieira, 1994) e está relacionada à

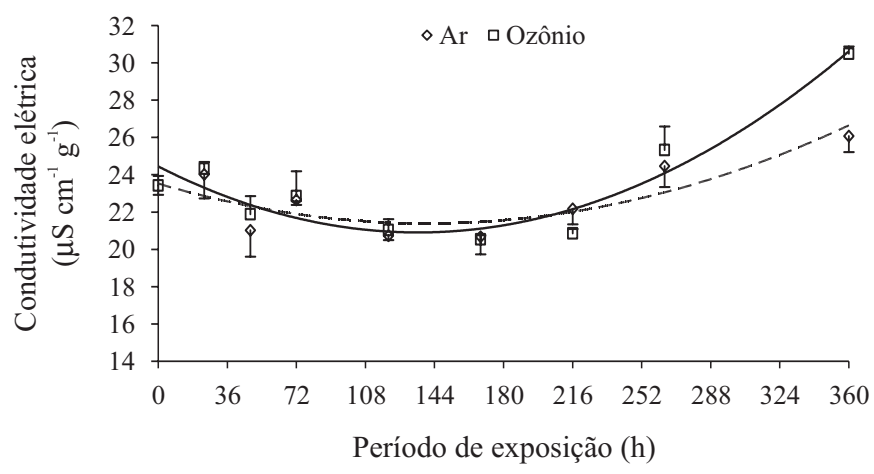

Figura 2. Condutividade elétrica da solução que contém os grãos de milho distribuídos na camada superior

Tabela 1. Médias das características fisiológicas avaliadas para os tratamentos com ozônio em relação aos tratamentos com ar atmosférico, distribuídos sobre o plenum e nas camadas mediana e superior da massa de grãos

\begin{tabular}{|c|c|c|c|c|c|c|}
\hline \multirow{2}{*}{ Análises } & \multicolumn{2}{|c|}{ Sobre o plenum } & \multicolumn{2}{|c|}{ Mediana } & \multicolumn{2}{|c|}{ Superior } \\
\hline & Ar & Ozônio & $\mathrm{Ar}$ & Ozônio & Ar & Ozônio \\
\hline $\begin{array}{l}\text { Teor de umidade } \\
\text { (\% b.u.) }\end{array}$ & $12,8 \pm 0,2$ & $12,7 \pm 0,2$ & $12,6 \pm 0,3$ & $12,6 \pm 0,2$ & $12,5 \pm 0,1$ & $12,5 \pm 0,1$ \\
\hline $\begin{array}{l}\text { Condutividade elétrica } \\
\qquad\left(\mu \mathrm{S} \mathrm{cm} \mathrm{cm}^{-1} \mathrm{~g}^{-1}\right)\end{array}$ & $22,3 \pm 1,4$ & $22,9 \pm 1,2$ & $22,3 \pm 1,1$ & $21,8 \pm 1,6$ & * & * \\
\hline $\begin{array}{c}\text { Germinação } \\
(\%)\end{array}$ & $68,4 \pm 2,9$ & $62,4 \pm 8,4$ & $67,7 \pm 3,5$ & $66,6 \pm 4,4$ & $70,5 \pm 5,1$ & $67,5 \pm 8,6$ \\
\hline
\end{tabular}

* Houve diferença significativa, portanto, representada na Figura 2 
deterioração dos mesmos, podendo-se inferir que grãos submetidos aos tratamentos com atmosfera modificada com $50 \mathrm{mg} \mathrm{kg}^{-1}$ de ozônio, em período superior a 11 dias consecutivos, pode causar uma redução moderada no seu valor qualitativo.

\section{CONCLUSÕES}

1. A eficácia no controle dos insetos-praga $S$. zeamais e T. castaneum é influenciada pelo período de exposição e pela distribuição dos insetos na massa de grãos, quando submetidos à atmosfera modificada com $50 \mathrm{mg} \mathrm{kg}^{-1}$ de ozônio.

2. O aumento do período de exposição resultou no aumento da eficácia dos tratamentos para os adultos de $S$. zeamais e $T$. castaneum.

3. A eficácia no controle de $S$. zeamais e $T$. castaneum reduziu à medida em que os insetos foram afastados do ponto de injeção do ozônio.

4. Em todos os tratamentos a espécie mais susceptível foi a S. zeamais, independentemente da sua distribuição na massa de grãos.

5. Os tratamentos com atmosfera modificada com 50 mg kg-1 de ozônio e com ar atmosférico, não afetaram a qualidade fisiológica dos grãos de milho.

\section{LITERATURA CITADA}

Brooker, D. B.; Bakker-Arkema, F. W.; Hall, C. W. Drying and storage of grains and oilseeds. New York: Van Nostrand Reinhold, 1992. 450p.

Kells, S. A.; Mason, L. J.; Maier, D. E.; Woloshuk, C. P. Efficacy and fumigation characteristics of ozone in stored maize. Journal of Stored Products Research, v.37, p.371-383, 2001.

Kim, J. G.; Yousef, A. E.; Dave, S. Application of ozone for enhancingthe microbiological safety and quality of foods: a review. Journal of Food Protection, v.62, p.1071-1087, 1999.

Mendez, F.; Maier, D. E.; Mason, L. J.; Woloshuk, C. P. Penetration of ozone into columns of stored grains and effects on chemical composition and performance. Journal of Stored Products Research, v.39, p.33-44, 2003.

Padin, S.; Bello, G. D.; Fabrizio, M. Grain loss caused by Tribolium castaneum, Sitophilus oryzae and Acanthoscelides obtectus in stored durum wheat and beans treated with Beauveria bassiana. Journal of Stored Products Research, v.38, p.69-74, 2002.

Rice, R. G.; Graham, D. M. Recent developments in food and agricultural uses of ozone, Annual Conference - Ozone Applications in a Changing Regulatory Environment. North Caroline: IOA- Raleigh, 2002, p.1-12.

Strait, C. A. Efficacy of ozone to control insects and fungi in stored grain. West Lafayette, Purdue University, 1998, 59 p. M.S. Thesis

Vieira, R. D. Teste de condutividade elétrica. In: Vieira, R. D.; Carvalho, N. M. Testes de vigor em sementes. Jaboticabal: FUNEP/UNESP, 1994, p.103-132. 